\title{
O PENSAMENTO IBERO-AMERICANO: CAMINHO PARA A INTERNACIONALIZAÇÃO
}

\author{
PENSAMIENTO IBEROAMERICANO: CAMINO HACIA LA \\ INTERNACIONALIZACIÓN
}

IBERO-AMERICAN THINKING: THE PATHWAY TO INTERNATIONALIZATION

\author{
Thaís Conte VARGAS ${ }^{1}$ \\ Maíra Darido da CUNHA ${ }^{2}$
}

RESUMO: A análise que aqui se apresenta busca recuperar a trajetória recente de um grupo de pesquisadores que têm se dedicado ao estudo sistemático da Educação ibero-americana. Embora centenas de pesquisas venham sendo desenvolvidas sobre a mesma temática, o grupo de pesquisa Cervantes nasceu, em 2006, pelo acordo de cooperação entre a Universidade Estadual Paulista e a Universidade de Alcalá de Henares, com o intuito de oferecer troca de conhecimento entre as duas instituições. Os impactos das novas tecnologias e o crescimento do grupo provocaram intensa modificação na dinâmica de atuação dos participantes e na forma como os investigadores passaram a produzir e divulgar as contribuições científicas elaboradas. A partir de 2012, as ações de internacionalização se fortaleceram: outras universidades latinoamericanos se articularam ao grupo; há uma profunda reestruturação da Revista IberoAmericana de Estudos de Educação. Em 2014, toda a produção intelectual produzida passa a ser difundida em formato digital.

PALAVRAS-CHAVE: Educação. Ibero-América. Internacionalização. Divulgação científica.

RESUMEN: El análisis que aqui se presenta pretende recuperar la trayectoria reciente de un grupo de investigadores que se han dedicado al estudio sistemático de la Educación iberoamericana. Aunque se han desarrollado centenares de investigaciones sobre el mismo tema, el grupo de investigación Cervantes nació en 2006 por el acuerdo de cooperación entre la Universidad Estadual Paulista y la Universidad de Alcalá de Henares, con el fin de ofrecer un intercambio de conocimientos entre ambas instituciones. Los impactos de las nuevas tecnologías y el crecimiento del grupo provocaron una intensa modificación en la dinámica de las acciones de los participantes y en la forma por la que los investigadores comenzaron a producir y divulgar las contribuciones científicas elaboradas. A partir de 2012, las acciones de internacionalización se han fortalecido: otras universidades latinoamericanas se han unido al grupo; hay una profunda reestructuración de la Revista Iberoamericana de Estudios en Educación. En 2014, se empezó a difundir en formato digital toda la producción intelectual producida.

\footnotetext{
${ }^{1}$ Universidade Estadual Paulista (UNESP), Araraquara - SP - Brasil. Doutoranda no Programa de Pós-graduação em Educação Escolar. ORCID: https://orcid.org/0000-0003-3054-5979. E-mail: thaiscontev@hotmail.com

${ }^{2}$ Faculdade de Bertioga (FABE), Bertioga - SP - Brasil. Docente nos Cursos de Graduação e Pós-Graduação. Doutora em Educação Escolar (UNESP). ORCID: https://orcid.org/0000-0002-8263-882X. E-mail: maira_darido@yahoo.com.br
}

RIAEE - Revista Ibero-Americana de Estudos em Educação, Araraquara, v. 15, n. esp. 4, p. 2543-2555, dez., 2020. e-ISSN: 1982-5587 
PALABRAS CLAVE: Educación. Iberoamérica. Internacionalización. Divulgación científica.

ABSTRACT: The analysis here presented seeks to bring forth the recent trajectory of a group of researchers who have dedicated themselves to the systematic study of Ibero-American Education. Although hundreds of research papers are being written on the same theme, the research group Cervantes was born, in 2006, due to the cooperation agreement between the Sao Paulo State University and the University of Alcalá de Henares, in order to offer a knowledge exchange between the two institutions. The impacts of new technologies and the growth of the group caused an intense change in the dynamics of the participants' performance and in the way, researchers started to produce and disseminate the scientific contributions elaborated. As of 2012, the internationalization actions were strengthened: other Latin American universities joined the group and there was a profound restructuring of the IberoAmerican Journal of Education Studies. In 2014, all intellectual production was made available in digital format.

KEYWORDS: Education. Ibero-America. Internationalization. Scientific divulgation.

\section{Introdução}

Um dos critérios que vêm sendo exigidos dos programas de pós-graduação brasileiros é a internacionalização: grupos de investigação que rompem as fronteiras nacionais para encontrar parceiros que dialoguem sobre temas relevantes, que tenham rebatimentos práticos na ciência que se pratica internamente. Em um desses esforços, a Universidade Estadual Paulista "Júlio de Mesquita Filho" (UNESP) - através da Faculdade de Ciências e Letras, Campus de Araraquara (FCLAr) - estabeleceu, em 2006, um acordo de cooperação com a Universidade de Alcalá de Henares (UAH), Espanha, com a finalidade de trocar experiências acadêmicas, científicas e técnicas em Educação, possibilitando intercâmbios; projetos de investigação e divulgação conjuntos; e realização de eventos.

Muitos conceitos se desenvolveram em torno da temática da internacionalização ${ }^{3}$ e vários foram os movimentos dos grupos de investigação brasileiros para elencar seus parceiros: no caso da Educação, os pesquisadores da FCLAr consideraram oportuna a aproximação SulSul, ou seja, com os países de língua portuguesa e espanhola. Vale a pena lembrar que as Agências de Financiamento e mesmo a UNESP, no caminho contrário, passaram a incentivar, cada vez mais, os acordos Norte-Sul, ou seja, aqueles que se estabeleceram, principalmente, com universidades bem posicionadas nos ranques internacionais, vale dizer, preferencialmente, com países de língua inglesa.

\footnotetext{
${ }^{3}$ Alguns aspectos da internacionalização são tratados por Bizelli e Santos-Cruz: os ambientes nos quais ela se dá (BIZELLI; SANTOS CRUZ, 2016); os impactos diretos na pós-graduação (BIZELLI, 2017); e seus medidores utilizados para valorar produção intelectual (BIZELLI; SANTOS CRUZ, 2019).
}

RIAEE - Revista Ibero-Americana de Estudos em Educação, Araraquara, v. 15, n. esp. 4, p. 2543-2555, dez., 2020. e-ISSN: 1982-5587 
Diante da opção feita, surge a parceria com a UAH, universidade com densidade acadêmica reconhecida internacionalmente e que vinha ampliando sua participação nos cursos de pós-graduação de vários países da América Latina. $\mathrm{O}$ acordo de cooperação - assinado em 2006 pelas duas universidades ${ }^{4}$ - previa a manutenção de encontros periódicos - programados para acontecer anualmente com alternância de sede entre as duas instituições -; o intercâmbio de pesquisadores - principalmente para programas de pós-doutorado -; e a sistemática produção e divulgação de ideias, conceitos e pesquisas sobre a educação escolar que vem sendo feita na Ibero-América.

Os Encontros Ibero-Americanos de Educação (EIDEs) passam a acontecer a partir de 2006, seguindo o modelo originalmente desenhado, mas, em 2012, foi a Universidade Metropolitana de Ciências da Educação, da cidade de Santiago do Chile, que sediou o evento, apoiada pela Fundação Creando Futuro. Embora nos anos ímpares os EIDEs continuassem a acontecer no Brasil, nos outros anos foram se agregando novas sedes em outros países: Colômbia, México e Peru (SEBASTIÁN HEREDERO; BIZELLI; MARTIN BRIS, 2018).

No entanto, foi nos veículos de difusão do conhecimento gerado pelo grupo que as mudanças se fizeram mais fortes. Para acompanhar as transformações do mercado editorial mundial, os Anais do EIDE; as obras publicadas; e a Revista Ibero-Americana de Estudos em Educação (RIAEE) passaram por uma reconfiguração completa, aumentando as chances de criar um campo internacionalizado de discussão para além dos pesquisadores diretamente envolvidos com as questões educativas e seus respectivos territórios nacionais, como será possível verificar neste artigo.

\section{Encontros, produtos e entornos internacionalizados}

As necessidades nascidas das exigências de organismos como a $\mathrm{CAPES}^{5}$ induziram a um modelo de avaliação da produção daqueles docentes e discentes que se dedicam à pósgraduação, nas últimas décadas. De certa forma, as agências de controle, focando na internacionalização, valoraram um conjunto de indicadores, parâmetros ou atividades: participação em eventos - nacionais e internacionais - e publicação conjunta - orientador e orientando - em veículos qualificados globalmente.

${ }^{4}$ Este acordo tem como unidades executoras a FCLAr, representando a UNESP, e o Departamento de Ciencias de la Educación, representando a UAH.

${ }^{5}$ O sistema de avaliação da pós-graduação brasileira se inicia em 1951, quando se cria a Coordenação de Aperfeiçoamento de Pessoal do Ensino Superior (Capes), fundação do Ministério da Educação (MEC).

RIAEE - Revista Ibero-Americana de Estudos em Educação, Araraquara, v. 15, n. esp. 4, p. 2543-2555, dez., 2020. e-ISSN: 1982-5587 
Segundo Barradas Barata (2016), após uma fase de avaliação incipiente, a CAPES estabeleceu, em 1990, um sistema de notas - de 1 a 5 - para classificar os programas de pósgraduação, sendo que, em 1998, ela introduziu a ficha de avaliação. No mesmo período, iniciase uma preocupação em medir a qualidade da produção de docentes através da atribuição de notas para os periódicos científicos. Permitia-se, assim, distinguir os artigos de professores através da importância das revistas nas quais eles publicavam. Com relação aos livros, o valor se dava pelo status da editora. Os extratos iniciais que classificavam as revistas foram reformulados em 2008 - passando a conter uma escala de 7 níveis - e em 2018 - passando a conter 8 níveis. É importante destacar, também, que as últimas reformulações na ficha de avaliação da Capes diminuíram o peso da avaliação docente - analisado por publicações - de 40\% (avaliação 2013-2016) para 12.5\% (avaliação atual, 2017-2020).

De qualquer forma, a corrida atual é para orientar todo movimento de valoração dos periódicos científicos por indicadores como Fator de Impacto, cujo parâmetro é o índice de citação - quantas vezes um texto é citado em determinadas bases de dados, normalmente Scopus e Web of Science - que atingem os artigos de um determinado veículo.

Descortinando os interesses que se ocultam em todas essas métricas que vêm sendo utilizadas, é possível perceber dois movimentos: um forte interesse privatista para manter o controle da produção científica internacional ${ }^{6}$ e o avanço das tecnologias de informação e comunicação para medir dentro dos dados produzidos pela Ciência. O primeiro movimento pode ser verificado, empiricamente, quando se constata que o mercado privado de indexadores é dominado por duas empresas privadas: a Elsevier - que produz o indicador SciVerse Scopus, ou simplesmente Scopus - e a Clarivate Analytics - que produz o indicador Web of Science.

O segundo movimento caracteriza-se pela construção histórica moderna do Big Data que abriga a produção científica mundial e do aperfeiçoamento de instrumentos digitais de mineração de informações que permitem extrair quantitativos desse universo de dados. Quanto mais os meios de armazenamento eletrônicos foram se desenvolvendo e aumentando sua capacidade quantitativa, mais eles abriram espaço para a circulação e compartilhamento do conhecimento. No momento em que as ferramentas de mineração passaram a organizar e fornecer relatórios por assunto, por palavras-chave, por autores e por veículos de difusão, os

${ }^{6} \mathrm{O}$ domínio das empresas citadas e o produtivismo acadêmico ocasionado pela disputa por atingir maior Fator de Impacto entre os pesquisadores e veículos científicos receberam muitas críticas, no Brasil (SGUISSARDI; SILVA JÚNIOR, 2009; BIANCHETTI; VALLE; PEREIRA, 2015; KUHLMANN JR, 2015), e até em organismos internacionais como entre os signatários da San Francisco Declaration on Research Assessment (DORA). Disponível em: http://www.ascb.org/dora/. Acesso em: 10 set. 2020.

RIAEE - Revista Ibero-Americana de Estudos em Educação, Araraquara, v. 15, n. esp. 4, p. 2543-2555, dez., 2020. e-ISSN: 1982-5587 
acadêmicos perderam o controle sobre sua própria produção e passaram a dialogar sem nenhum tipo de barreira, nem mesmo a linguística.

O que passa a ocorrer com os encontros e congressos internacionais segue a mesma lógica, já que o resultado dessa mobilidade internacional é passível de ser concretizado nos relatórios e depositórios que guardam a memória do evento. Mais uma vez, o material sistematizado e transformado em arquivos de texto pode circular dentro das bases do Big Data, através de atas ou anais de atividades realizadas em diversos países, mas também através de livros que circulem na comunidade científica global. Assim, é possível pensar, por exemplo, na formação de uma escola ibero-americana de pensamento sobre a Educação, ou seja, de um grupo de pesquisadores internacionais que se dedicam a estudar a educação escolar no território ibero-americano.

\section{A RIAEE: novos patamares bibliográficos}

De forma simplificada, a RIAEE passou por quatro fases que poderiam ser definidas como: inicial; aprimoramento; consolidação acadêmica; e reconhecimento científico. A primeira fase vai da data de criação da revista (2006) a 2008, quando o veículo tinha regularidade semestral e trazia, fundamentalmente, contribuições de autores vinculados à UNESP e à UAH. Entre 2009 e 2011, ela passa a ter três edições por ano e começa a se abrir para novos autores.

Em 2012, começa a transição do periódico para um novo modelo: as edições são trimestrais; os autores são diversificados segundo sua origem acadêmica; sua classificação na CAPES passou a ser B1. Entre 2015 e os dias atuais, a RIAEE ganha edições especiais; incrementa positivamente seus indexadores; evita a endogenia de autores; diversifica seu Conselho Editorial Nacional e Internacional, com intelectuais e acadêmicos respeitados em diversos países; e recebe o conceito A2, em Educação, na CAPES (quadriênio 2013-2016, mantido em 2019). O quadro abaixo mostra mudanças numéricas, ocorridas a partir de 2014. 
Tabela 01 - Dados de itens publicados pela RIAEE: 2014- 2020

\begin{tabular}{|c|c|c|c|}
\hline Ano & Número de Edições & $\begin{array}{c}\text { Número de } \\
\text { Artigos }\end{array}$ & $\begin{array}{c}\text { Número de } \\
\text { páginas }\end{array}$ \\
\hline 2014 & 4 regulares & 79 & 1034 \\
\hline 2015 & 4 regulares e 2 especiais & 105 & 1686 \\
\hline 2016 & 4 regulares e 4 especiais & 169 & 2593 \\
\hline 2017 & 4 regulares e 2 especiais & 149 & 2291 \\
\hline 2018 & 4 regulares e 3 especiais & 128 & 2099 \\
\hline 2019 & 4 regulares e 4 especiais & 144 & 2103 \\
\hline $2020^{*}$ & 4 regulares e 3 especiais* & $124 *$ & $2145^{*}$ \\
\hline Totais & 46 edições & $\mathbf{8 9 8}$ & $\mathbf{1 3 9 5 1}$ \\
\hline
\end{tabular}

*Dados referentes a outubro de 2020

Fonte: Elaborado pelas autoras

A RIAEE é publicada na base SEER (Sistema Eletrônico de Editoração de Revistas), mantém suas edições no formato XML e divulga-as pelo Directory of Open Access Journals ${ }^{7}$ (DOAJ). Da mesma forma, a revista está disponível em outras bases de dados nacionais - como Diadorim, Portal de Periódicos CAPES, Edubase, BBE, Sumários.org, Cnen/Livre Portal de Periódicos - e internacionais - como Web of Science; Ebsco Host; Redalyc; Latindex; Clase; Iresie; ErihPlus; Dialnet; Google Scholar; Redib; Miar/Universidad de Barcelona; entre outras.

Recuperando a discussão sobre a capacidade que os veículos científicos necessitam para transitar no Big Data, é possível perceber, na Figura 01 apresentada abaixo, que o impacto da RIAEE nos últimos 8 anos é crescente, tanto no número de citações como nos indicadores do índice $h$ ou mesmo no $h 10$.

Apreende-se, então, em 2016, que a RIAEE recebeu 158 citações; em 2017, 290; em 2018, 415; 2019, 562; e, em 2020, até outubro, 575 citações. Os índices $h$ dizem respeito ao número $h$ de artigos que foram citados $h$ vezes, em um determinado período. Exemplo: o $h 10$ são os 10 artigos que foram citados 10 vezes em período determinado, no caso da RIAEE nos últimos anos, de 2005 até agora, o índice $h 10$ é 29.

${ }^{7}$ A base internacional de dados DOAJ foi criada em 2003 pela Universidade de Lund, Suécia, e abriga revistas acadêmicas e científicas de acesso aberto, que possuam revisão por pares. Ampliar o impacto de periódicos é sua missão, aumentando a visibilidade e facilitando o uso de revistas acadêmicas.

RIAEE - Revista Ibero-Americana de Estudos em Educação, Araraquara, v. 15, n. esp. 4, p. 2543-2555, dez., 2020. e-ISSN: 1982-5587 
Figura 01 - Dados bibliométricos da RIAEE no Google Scholar

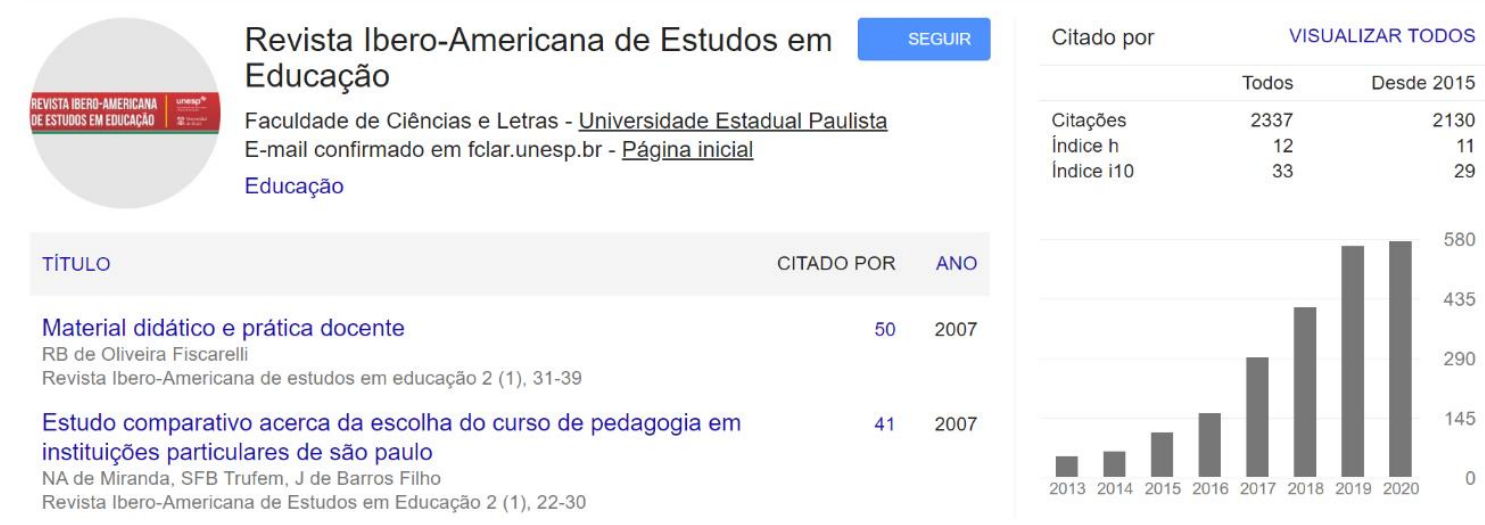

Fonte: Google Scholar

A RIAEE torna-se, portanto, um dos principais espaços de discussão crítica do grupo que se estruturou em torno à UNESP, à UAH e aos demais investigadores do Brasil, da Espanha e dos demais países da Ibero América. Assim, também, modificam-se a qualidade das trocas presenciais ou digitais - que configuraram os EIDEs e as obras produzidas pelos pesquisadores da Educação Escolar ibero-americana.

\section{Os EIDEs e a circulação digital de seus produtos}

É importante notar que os EIDEs reuniram, em todas suas versões, um conjunto de público e trabalhos acadêmicos significativos. No entanto, aponta-se que há um profundo descompasso quantitativo entre os eventos que acontecem no Brasil e aqueles que se dão em outros países. A cultura de participação em eventos fora do território nacional é muito diferente: os eventos são mais focados por temáticas específicas; as inscrições e despesas são relativamente mais elevadas e não contam com financiamentos de Agências de Fomento; não há um incentivo institucional para participação de estudantes, transformando as sessões de apresentação em espaços de aprofundamentos entre especialistas.

As figuras 02 e 03 comparam a participação no evento dentro e fora do Brasil, indicando o número de trabalhos inscritos em cada EIDE. Assim, é possível perceber os traços indicados anteriormente. 
Figura 02 - Participantes e trabalhos apresentados nos EIDEs ocorridos no Brasil

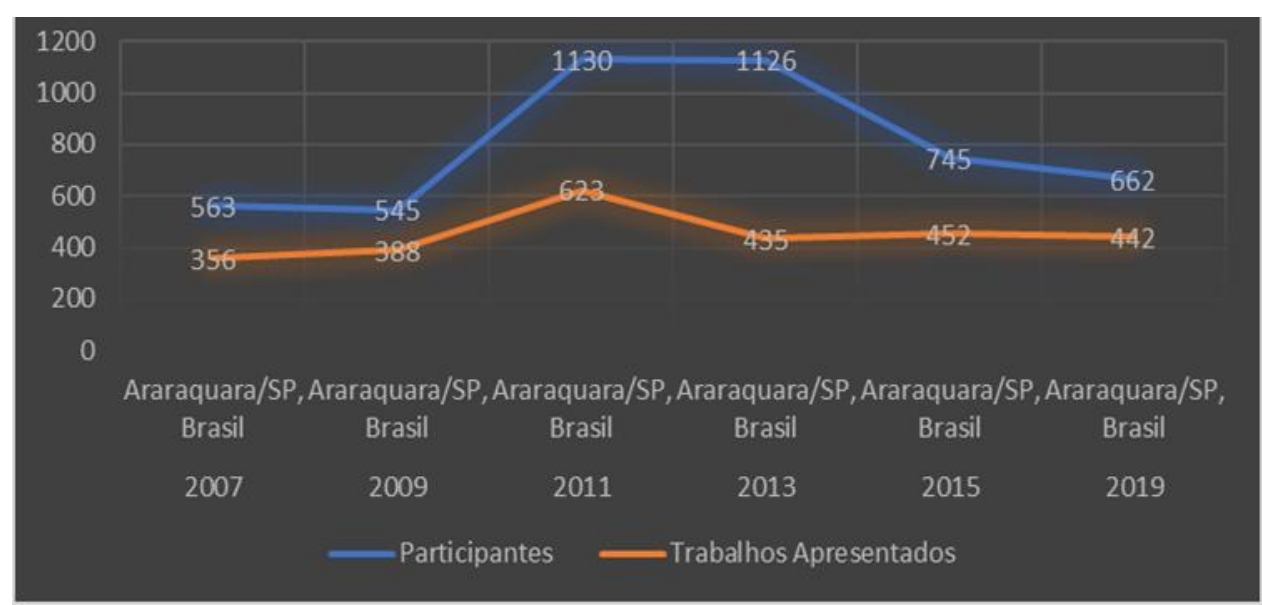

Fonte: Elaborado pelas autoras

Figura 03 - Participantes e trabalhos apresentados nos EIDEs ocorridos no exterior

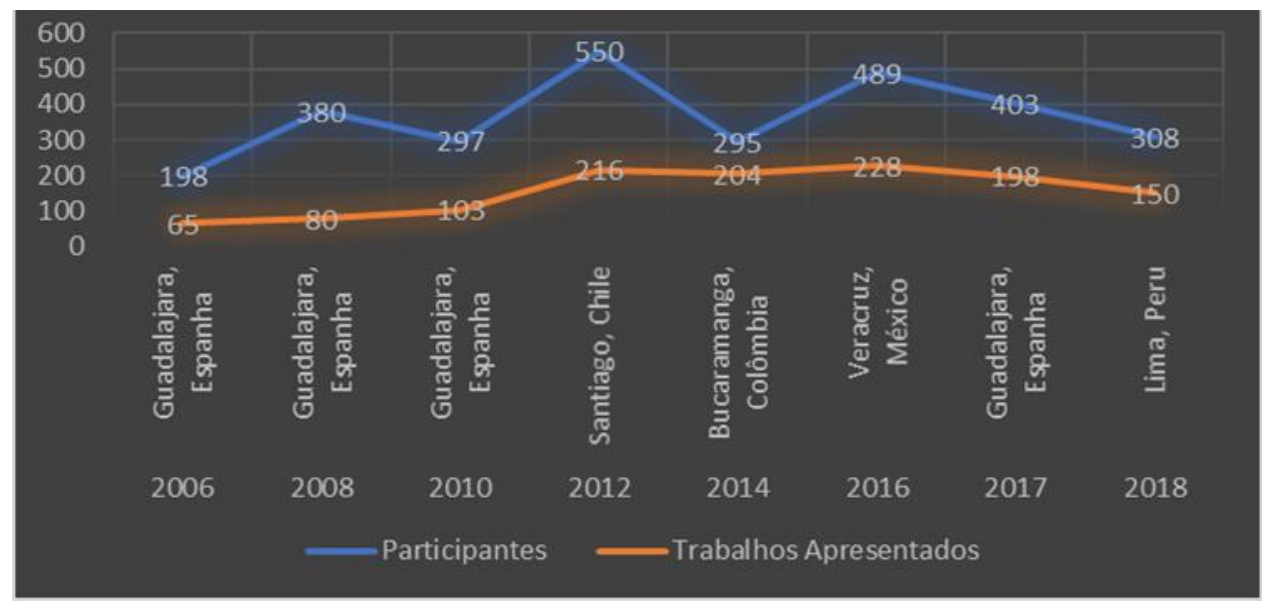

Fonte: Elaborado pelas autoras

Conforme expresso anteriormente, este artigo se centra nas mudanças de veículos de difusão dos resultados dos EIDEs e das publicações elaboradas pelo grupo. Sendo assim, por um lado, a partir de 2014, as Memórias - ou Actas, ou Anais - do EIDE passaram a ser trabalhadas através de projeto editorial, com DOI - além do tradicional ISBN -, e amplamente divulgadas nos meios digitais ${ }^{8}$, como pode ser visto nas obras coordenadas por Martinez, Osorio e Acosta (2015); Bizelli, Muzzeti e Souza Lemes (2016); Bizelli, Heredero, Vargas e Santos Cruz (2019).

Por outro lado, todos os EIDEs foram precedidos de material de divulgação digital, reportando os resultados de pesquisa ou os debates intelectuais, nos diferentes países sobre os

${ }^{8}$ No ano de 2016, os trabalhos apresentados em Veracruz, México, forma articulados em cinco livros organizados por Miranda-Alvarez e Edel-Navarro $\left(2016^{(1)}, 2016^{(2)}, 2016^{(3)}, 2016^{(4)}, 2016^{(5)}\right)$.

RIAEE - Revista Ibero-Americana de Estudos em Educação, Araraquara, v. 15, n. esp. 4, p. 2543-2555, dez., 2020. e-ISSN: 1982-5587 
temas que dizem respeito à Educação Escolar em Ibero América. Novamente aqui, os livros físicos foram sendo substituídos pelos eletrônicos, como nos exemplos: Bizelli e Souza (2014 ${ }^{(1)}$, 2014 ${ }^{(2)}$ ); Jabonero, Martín Bris, Arias e Bizelli (2015); Bizelli, Heredero e Ribeiro (2015); Martín Bris, Miranda-Álvarez, Sebastián-Heredero e Edel Navarro $\left(2016^{(1)}, 2016^{(2)}, 2016^{(3)}\right)$; Martín-Bris e Sebastián-Heredero (2017); Acevedo Tovar (2018); Bizelli e Muzzeti (2019 $9^{(1)}$, $\left.2019^{(2)}, 2020\right)$.

São doze livros digitais em seis anos, publicados por editoras com selos conceituados, tanto no Brasil - a exemplo do selo Cultura Acadêmica da Editora da UNESP -, como na Espanha - a exemplo das editoras da UAH e Santillana -, e na América Latina - a exemplo das editoras das Universidades de Veracruz (México) e Nacional Mayor de San Marcos (Peru). Consolida-se, portanto, um grupo de autores que impulsionam os debates sobre a educação escolar ibero-americana - em torno de trinta a quarenta intelectuais que trabalham de forma mais próxima - e um conjunto de mais de 100 pesquisadores que gravitam ao redor deste núcleo.

\section{Considerações finais}

Conforme foi mostrado neste artigo, os esforços de internacionalização promovidos por universidades brasileiras produziram alianças estratégicas com grupos internacionais de investigação. Embora tenha havido um forte incentivo para as articulações Norte-Sul principalmente com países de língua inglesa que ocupam altas posições em ranques acadêmicos -, o Grupo Cervantes articulou suas ações, a partir de 2006, focando a educação escolar que vem sendo feita na Ibero-América. Formado inicialmente com pesquisadores da UNESP e da UAH, em 2012, o grupo passa a deslocar seus encontros anuais para outros países da América Latina.

O centro da análise realizada diz respeito à transformação que vai se processando, depois de 2014, nos meios utilizados pelo grupo para divulgar suas contribuições. Um primeiro caminho se faz através da revista criada (RIAEE), que passa a adotar padrões de indexação e de comunicação com o Big Data digital - através de sua versão em linguagem $x m l$. Um segundo caminho impacta as obras produzidas: tanto aquelas que preparam anualmente os EIDEs, como as que apresentam seus resultados.

Os dados que se mostram mais claros, portanto, são os que estão relacionados ao desempenho obtido pela RIAEE, nos quais é possível verificar crescimento quantitativo número de citações no google scholar - e crescimento qualitativo - variedade de artigos 
nacionais e internacionais, indexação em bases importantes, baixa endogenia entre autores que participam do grupo. No entanto, é preciso notar também que, no último quinquênio, foram produzidos, em média, dois livros digitais por ano, o que demonstra que os pesquisadores vinculados ao grupo se constituem enquanto referência na temática proposta.

\section{REFERÊNCIAS}

ACEVEDO TOVAR, L. M. (Org.). Innovación e interculturalidad en la educación. 1. ed. Lima: Fondo Editorial de la Universidad Nacional Mayor de San Marcos/Facultad de Educación, 2018. Disponível em: http://iage.fclar.unesp.br/eide/arqs/eide2018.pdf. Acesso em: 5 nov. 2020.

BARRADAS BARATA, R. C. Dez coisas que você deveria saber sobre o Qualis. Revista Brasileira de Pós-Graduação, v. 13, n. 30, 22 dez. 2016. DOI: https://doi.org/10.21713/2358-2332.2016.v13.947

BIANCHETTI, L.; VALLE, I. R.; PEREIRA, G. R. M. O fim dos intelectuais acadêmicos? Induções da Capes e desafios às associações científicas. Campinas, SP: Autores Associados, 2015.

BIZEL BIZELLI, J. L.; MUZZETI, L. R. (Org.). Educação e formação de professores. 1. ed. Itapetininga, SP: Ed. Hipótese, 2019(2). Disponível em: https://251c5e01-0d17-4728-99f40ed664f18de0.filesusr.com/ugd/c8e391_4b18c29dcb1845079ac044f5919305c6.pdf?index=tr ue. Acesso em: 5 nov. 2020.

BIZEL BIZELLI, J. L.; MUZZETI, L. R. (Org.). Educação e trabalho docente. 1. ed. Bauru, SP: Editora Ibero-americana de Educação, 2020. Disponível em: https://251c5e01-0d17-472899f4-0ed664f18de0.filesusr.com/ugd/c8e391_57d5a982fddd4191a46bf3e8f4fac2d9.pdf.

Acesso em: 5 nov. 2020.

BIZELLI, J. L. Internacionalización: reflexiones a partir de la experiencia de un programa de postgrado en Educación Escolar brasileña. In: MARTÍN BRIS, M; JABONERO BLANCO, M. (Org.). Internacionalización de la educación en Iberoamérica: reflexiones y proyecciones. 1. ed. Alcalá de Henares: Universidad de Alcalá/Editora Santillana, 2017. v. 1. p. 35-41.

BIZELLI, J. L.; HEREDERO, E. S.; RIBEIRO, P. R. M. (Org.). Inclusão e aprendizagem: desafios para a escola em Ibero-América. 1. ed. São Paulo: Cultura Acadêmica, 2015. Disponível em:

https://www.fclar.unesp.br/Home/Instituicao/Administracao/DivisaoTecnicaAcademica/Apoi oaoEnsino/LaboratorioEditorial/serie-temas-em-educacao-escolar-n23.pdf. Acesso em: 5 nov. 2020.

BIZELLI, J. L.; HEREDERO, E. S.; VARGAS, T. C.; SANTOS CRUZ, J. A. ENCONTRO IBERO AMERICANO DE EDUCAÇÃO, 14., 2019, Araraquara. Anais [...]. Araraquara, SP: UNESP; Bauru, SP: Editora Ibero-Americana de Educação, 2019. Disponível em: https://www.editoraiberoamericana.com/anaisxiveide2019. Acesso em: 5 nov. 2020. 
BIZELLI, J. L.; MUZZETI, L. R. (Org.). Práticas pedagógicas em educação. 1. ed. Itapetininga: Edições Hipótese, 2019(1). Disponível em: https://251c5e01-0d17-4728-99f40ed664f18de0.filesusr.com/ugd/c8e391_5f7709d1ca1343aaaa00cb3126adc920.pdf?index=tru e. Acesso em: 5 nov. 2020.

BIZELLI, J. L.; SANTOS CRUZ, J. A. Educación e Internacionalización: ¿competencia o colaboración? In: MARTIN BRIS, M. (Org.). Internacionalización de la educación superior en Iberoamérica: miradas y perspectivas. 1. ed. Alcalá de Henares: Universidad de Alcalá, 2016. v. 1. p. 77-90.

BIZELLI, J. L.; SANTOS CRUZ, J. A. Indexadores internacionais para medir a produção docente na área de educação. In: LAMOTHE ZAVALETA, C.; MIRANDA ÁLVAREZ, M. C.; MARTÍN BRIS, M. (Org.). Práctica docente: experiencias, reflexiones y propuestas desde Iberoamérica. 1. ed. Veracruz: Universidad Veracruzana/México Academia Journals, 2019. v. 1. p. 137-148.

BIZELLI, J. L.; SOUZA, C. B. G. (Org.). Caminhos para a Escola Inclusiva. 1. ed. São Paulo: Cultura Acadêmica, 2014 ${ }^{(2)}$. Disponível em: https://www.fclar.unesp.br/Home/Instituicao/Administracao/DivisaoTecnicaAcademica/Apoi oaoEnsino/LaboratorioEditorial/serie-temas-em-educacao-escolar-n.21.pdf. Acesso em: 5 nov. 2020.

BIZELLI, J. L.; SOUZA, C. B. G. (Org.). Faces da escola em ibero-américa. 1. ed. São Paulo: Cultura Acadêmica, 2014 ${ }^{(1)}$. Disponível em:

https://www.fclar.unesp.br/Home/Instituicao/Administracao/DivisaoTecnicaAcademica/Apoi oaoEnsino/LaboratorioEditorial/serie-temas-em-educacao-escolar-n.20.pdf. Acesso em: 5 nov. 2020.

BIZELLI; J. L.; MUZZETI, L. R.; SOUZA LEMES, S. ENCONTRO IBERO-AMERICANO DE EDUCAÇÃO, 5., 2016, Araraquara. Anais [...]. Araraquara, SP: UNESP, 2016. Disponível em: http://iage.fclar.unesp.br/eide/arqs/eidex.pdf. Acesso em: 5 nov. 2020.

JABONERO, M.; BRIS, M. M.; ARIAS, A. M.; BIZELLI, J. L. (Org.). Miradas diversas de la educación en Iberoamérica. 2. ed. revisada e ampliada. Alcalá de Henares, Espanha: Universidad de Alcalá, 2015. Disponível em:

https://ebuah.uah.es/dspace/bitstream/handle/10017/21398/9788415834946.pdf?sequence=1\& isAllowed=y. Acesso em: 5 nov. 2020.

KUHLMANN JR., Moysés. Produtivismo acadêmico, publicação em periódicos e qualidade das pesquisas. Caderno de Pesquisa, v. 45, n. 158, p. 838-855, 2015. DOI: https://doi.org/10.1590/198053143597

MARTÍN BRIS, M.; MIRANDA ÁLVAREZ, M. C.; SEBASTIÁN HEREDERO, E.; EDEL NAVARRO, R. (Org.). Visiones de la educación en Iberoamérica: experiencias y reflexiones. Tomo I. La educación para todos y la calidad. 1. ed. Veracruz, México: Universidad Veracruzana, 2016 $6^{(1)}$. v. 1. Disponível em:

https://www.uv.mx/veracruz/eide/memorias-del-encuentro/. Acesso em: 5 nov. 2020. 
MARTÍN BRIS, M.; MIRANDA ÁLVAREZ, M. C.; SEBASTIÁN HEREDERO, E.; EDEL NAVARRO, R. (Org.). Visiones de la educación en Iberoamérica: experiencias y reflexiones. Tomo II. Tópicos emergentes de la educación. 1. ed. Veracruz, México: Universidad Veracruzana, $2016^{(2)}$. v. 2. Disponível em:

https://www.uv.mx/veracruz/eide/memorias-del-encuentro/. Acesso em: 5 nov. 2020.

MARTÍN BRIS, M.; MIRANDA ÁLVAREZ, M. C.; SEBASTIÁN HEREDERO, E.; EDEL NAVARRO, R. (Org.). Visiones de la educación en Iberoamérica: experiencias y reflexiones. Tomo III. Una Mirada a la pluralidad educativa. 1. ed. Veracruz, México: Universidad Veracruzana, 2016 ${ }^{(3)}$. v. 3. Disponível em:

https://www.uv.mx/veracruz/eide/memorias-del-encuentro/. Acesso em: 5 nov. 2020.

MARTÍN BRIS, M.; SEBASTIÁN HEREDERO, E. (Org.). Hacia un modelo educativo de calidad y transformador. 1. ed. Madrid: Ed Santillana/Universidad de Alcalá, 2017.

Disponível em:

http://www.redage.org/sites/default/files/adjuntos/hacia_un_modelo_educativo.pdf. Acesso em: 5 nov. 2020.

MARTINEZ, A.; OSORIO, L. M.; ACOSTA, J. Reflexiones sobre la Educacion en Iberoamerica: Memorias del IX Encuentro Iberoamericano de Educación. Colombia, Bucaramanga: UNAB, 2015. Disponível em: http://iage.fclar.unesp.br/eide/arqs/eideix.pdf. Acesso em: 5 nov. 2020.

MIRANDA ALVAREZ, M. C.; EDEL NAVARRO, R. (Org.). Educación, tecnología e innovación: la tríada indisoluble. Veracruz, México: Universidad Veracruzana, $2016^{(1)}$. Disponível em: https://www.uv.mx/veracruz/eide/memorias-del-encuentro/. Acesso em: 5 nov. 2020.

MIRANDA ALVAREZ, M. C.; EDEL NAVARRO, R. (Org.). La Diversidad: encuentro y análisis. Veracruz, México: Universidad Veracruzana, 2016 ${ }^{(3)}$. Disponível em:

https://www.uv.mx/veracruz/eide/memorias-del-encuentro/. Acesso em: 5 nov. 2020.

MIRANDA ALVAREZ, M. C.; EDEL NAVARRO, R. (Org.). La Educación Básica: vivencias y reflexiones para contar. Veracruz, México: Universidad Veracruzana, 2016 ${ }^{(4)}$. Disponível em: https://www.uv.mx/veracruz/eide/memorias-del-encuentro/. Acesso em: 5 nov. 2020.

MIRANDA AlVAREZ, M. C.; EDEL NAVARRO, R. (Org.). Perspectivas e Identidades en el contexto de la Política Educativa. Veracruz, México: Universidad Veracruzana, $2016^{(5)}$, p. 14-19. Disponível em: https://www.uv.mx/veracruz/eide/memorias-del-encuentro/. Acesso em: 5 nov. 2020.

MIRANDA ALVAREZ, M. C.; EDEL NAVARRO, R. (Org.). Perspectivas, visiones e abordajes en Educación Superior. Veracruz, México: Universidad Veracruzana, 2016 $6^{(2)}$. Disponível em: https://www.uv.mx/veracruz/eide/memorias-del-encuentro/. Acesso em: 5 nov. 2020.

SEBASTIÁN HEREDERO, E.; BIZELLI, J. L.; MARTIN BRIS, M. Un recorrido por los encuentros iberoamericanos de educación. Una historia de producción científica

RIAEE - Revista Ibero-Americana de Estudos em Educação, Araraquara, v. 15, n. esp. 4, p. 2543-2555, dez., 2020. e-ISSN: 1982-5587 
interinstitucional consolidada. Revista Tendencias Pedagógicas, Madrid, v. 30, p. 329-338, 2017.

SGUISSARDI, V.; SILVA JÚNIOR, J. R. Trabalho Intensificado nas federais: pósgraduação e produtivismo acadêmico. São Paulo: Xamã, 2009.

\section{Como referenciar este artigo}

VARGAS, T. C.; CUNHA, M. D. O pensamento ibero-americano: caminho para a internacionalização. Revista Ibero-Americana de Estudos em Educação, Araraquara, v. 15, n. esp. 4, p. 2543-2555, dez., 2020. e-ISSN: 1982-5587. DOI: https://doi.org/10.21723/riaee.v15iesp4.14503

Submetido em: 10/09/2019

Revisões requeridas: 10/01/2020

Aprovado em: 30/04/2020

Publicado em: 01/12/2020 